Mini Review

\title{
Precancerous Lesions in the Large Intestine of Rodents
}

\author{
Hideki Mori ${ }^{1}$ and Yasuhiro Yamada ${ }^{1}$ \\ ${ }^{1}$ Department of Pathology, Gifu University School of Medicine, 40 Tsukasa-machi, Gifu 500-8705, Japan
}

\begin{abstract}
As precancerous lesions for large bowel cancers, early appearing lesions like aberrant crypt foci (ACF) are recognized. Recently, we identified $\beta$-cetenin accumulated crypts (BCAC) in the colonic mucosa of rats given a colon carcinogen. $\mathrm{BCAC}$ have frequent $\beta$-catenin gene mutations and their pathological features are different from those of ACF. Further comparative studies on the molecular pathology and biology of both lesions, gave rise to evidences that $\mathrm{BCAC}$ are probably the direct precursor for the large intestinal cancers in rodents. The newly identified lesions are suggested to be a reliable biomarker for the risk assessment of environmental chemicals and for the screening of cancer preventing agents. (J Toxicol Pathol 2002; 15: 129-132)
\end{abstract}

Key words: large bowel cancer, early appearing lesions, aberrant crypt foci, $\beta$-catenin-accumulated crypts, rodent

\section{Introduction}

The concept of precancerous lesions is going to be clear in certain organs of experimental animals as well as humans. Enzyme-alterted liver cell foci that appear in the process of rodent hepatocarcinogenesis is an example. Aberrant crypt foci (ACF) (Fig. 1) were first described by Bird in methylene blue-stained whole-mount preparations, of colon in animals treated with colon-specific carcinogens ${ }^{1,2}$. Numerous studies including molecular analysis have focused on the significance of ACF as early events in colon carcinogenesis, and the lesions are now regarded as putative precancerous lesions for colorectal cancers ${ }^{3,4}$. Similar lesions have been also identified in human colonic mucosa ${ }^{5}$. ACF are now used as one of useful biomarkers for evaluation of potential chemopreventive agents ${ }^{6}$. It is reported that number of crypts / focus of ACF increases with time after the carcinogen treatment, and that $\mathrm{ACF}$ have an increased proliferative activity 7,8 . Nevertheless, there are evidences that document lack of correlation between tumor development and expression of ACF. Furthermore, it is also known that several compounds with potency to prevent the occurrence of ACF, e.g., 2-(carboxyphenyl)retinamide or genistein, enhance the development of colon cancers ${ }^{9,10}$. Thus, it must be said that precancerous nature of ACF in the colon carcinogenesis still remains inconclusive. Recently, the authors identified $\beta$-catenin-accumulated crypts (BCAC)

Received: 10 April 2002, Accepted: 27 April 2002

Mailing address: Hideki Mori, Department of Pathology, Gifu

University School of Medicine, 40 Tsukasa-machi, Gifu 500-8705,

Japan

TEL: 81-58-267-2234 FAX: 81-58-265-9005

E-mail: hidmori@cc.gifu-u.ac.jp in the initial stage of colon carcinogenesis in rats ${ }^{11,12}$. The lesions have a number of different pathological properties from those of ACF, suggesting that BCAC are a direct precursor lesion for colorectal neoplasms ${ }^{11,12}$. Presently, the authors review of precancerous lesions for large intestinal cancers in rodents with emphasis on such newly identified lesions.

\section{Pathology and Molecular Biology of BCAC}

BCAC are recognized by analysis in en face preparations and in serial sections after the observation in whole-mount preparations of the colonic mucosa ${ }^{11}$ (Fig. 1). The number of the crypts / lesion and histological abnormality of BCAC significantly increase with time course in a manner not like that of ACF. Cell proliferative activity of $\mathrm{BCAC}$ is higher than of $\mathrm{ACF}^{12}$. Interestingly, $\mathrm{BCAC}$ are frequently accompanied by Paneth cells. It is true that Paneth cells are rather frequently recognized in colonic tumors although they are rarely seen in normal colonic epithelium. Therefore, it is suggested that the participation of Paneth cells in BCAC indicates a dis-differentiating potential of such "dysplatic" crypts. It has been reported that putative preneoplastic lesions of rat colon cancers have decreased hexosaminidase activity ${ }^{13}$. Our results indicate that both of ACF and BCAC possess decreased activity of this enzyme although $\beta$-catenin accumulation is present only at $\mathrm{BCAC}^{12}$. $\mathrm{BCAC}$ have accumulation of $\beta$-catenin protein in the cytoplasm as well as in the nuclei ${ }^{11,12}$ (Fig. 1). In our previous study, $\beta$-catenin gene mutations were found in 10 out $15 \mathrm{BCAC}$, although such mutation was recognized only in 3 of $15 \mathrm{ACF}$. Mutation of $\beta$-catenin gene in BCAC is usually recognized in codons $28,30,32,34$, and $41^{11}$. Very recently, we conducted a study to compare types of the $\beta$ - 

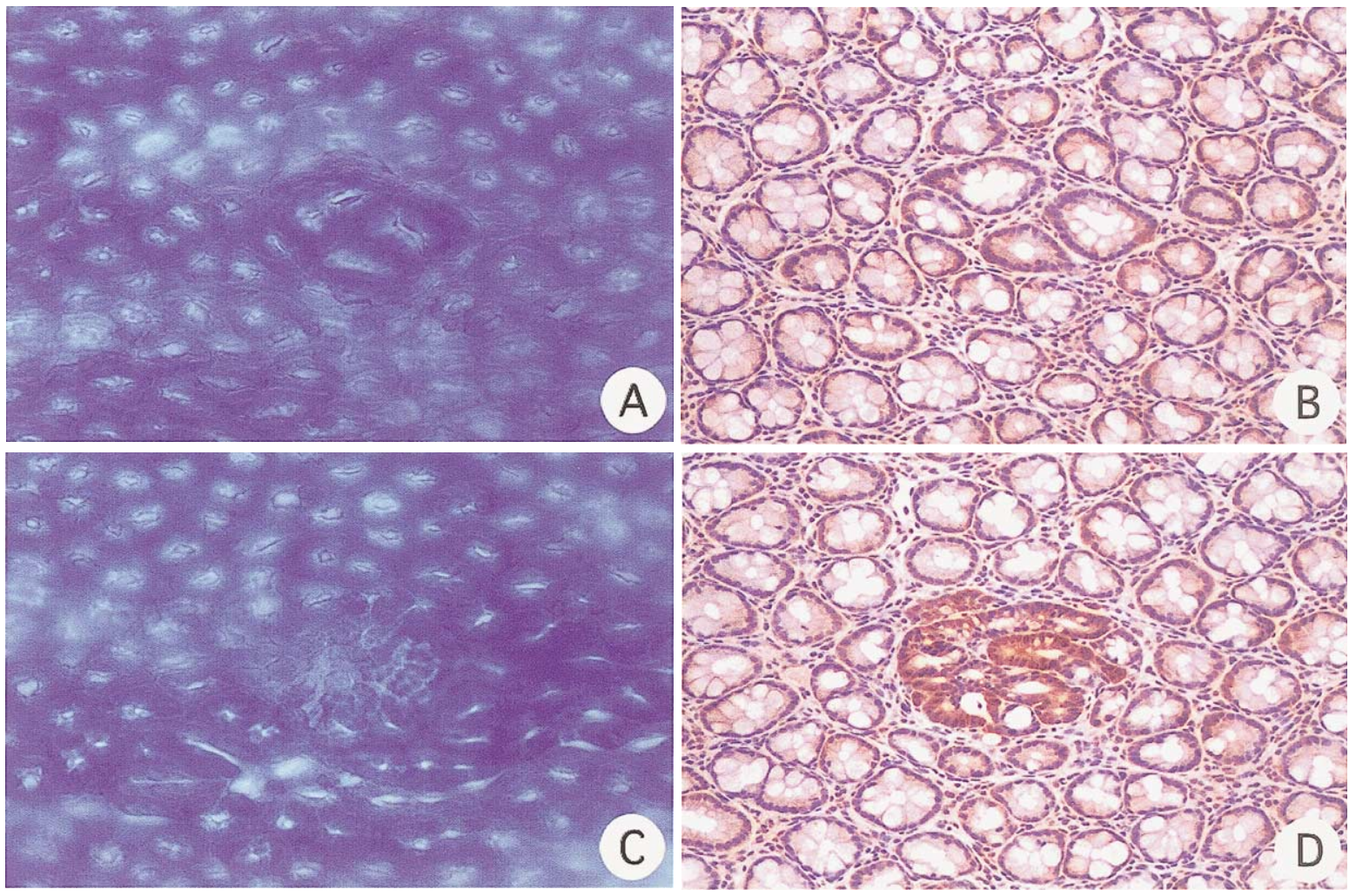

Fig. 1. Topographic view of an aberrant crypt focus in whole-mount preparation stained with methylene blue (A). $\beta$-Catenin immunohistochemistry in histological section of the corresponding area of $\mathrm{B}$. No accumulation of $\beta$-catenin protein is present (B). Topographic view of an equivocal lesion (center) without structure of ACF in whole-mount preparation stained with methylene blue (C). $\beta$-Catenin immunohistochemistry of the corresponding area of C. Accumulation of $\beta$ catenin protein is present $(\mathrm{D})$.

catenin and k-ras mutations of $\mathrm{BCAC}$ with those of colon tumors. Mutations in the exon 3 of $\beta$-catenin gene were respectively detected in $39.3 \%$ of BCAC and $56.8 \%$ of colon cancers. Remarkably, all $\beta$-catenin mutations recognized in the colon tumors converged at codons encoding functionally important residues that may directly mediate $\beta$-catenin degradation, whereas mutations in the early appearing lesions laid scattered in the exon 3 of the gene. In this study, frequency of k-ras mutations was rather higher than in colon cancers (unpublished results). Recently, target genes of the $\beta$-catenin-Tcf pathway were determined to be growthpromoting genes, such as c-myc and cyclin D $1^{14,15}$. It is also known that $\beta$-catenin levels are regulated by degradation of the protein via ubiquitin-protease pathway, and intact APC and AXIN cooperate with GSK- $3 \beta$ to regulate the degradation $^{16,17}$. Accordingly, our recent evidences suggest that broad spectrum of mutations are selected during the malignant transformation of the colon, and the main selective factor will be an activation of the $\beta$-catenin downregulating function. It is also implied that activation of $\beta$ catenin signaling pathway is not only an initiating event, but also plays a pivotal role in the promotion stage of colorectal carcinogenesis.

\section{Modification of Expression of BCAC}

Suppression of occurrence and advancement of premalignant lesions is important for cancer prevention. Effects of a selective cyclooxygenase-2 inhibitor, celecoxib which chemopreventive effect on the large bowel carcinogenesis has been confirmed in animal models ${ }^{18,19}$, on the development of $\mathrm{BCAC}$ in comparison with those of $\mathrm{ACF}$ was examined by us ${ }^{19}$. In this study, expression of BCAC was suppressed by exposure of celecoxib, and the suppression at BCAC was much more stronger than at ACF. Similarly, crypt multiplicity of BCAC was decreased by celecoxib and the decrease was stronger than at ACF. Numbers of silver stained nucleolar organizer regions / nucleus in BCAC were also decreased by celecoxib ${ }^{20}$. These data represent additional evidences that $\mathrm{BCAC}$ are premalignant lesions of colon cancer and suggest that chemopreventive effect of celecoxib is related to the decrease of expression of premalignant lesions as well as modulation of cell proliferation in the early appearing lesions.

It is considered that tumor promoters which enhance development of neoplasms also enhance the development of early appearing precancerous lesions in the target organs. 
Magnusen and $\operatorname{Bird}^{21}$, however, reported that cholic acid, a tumor promoter for colon cancers, did not enhance the occurrence of ACF but suppressed the expression of the lesions. Modulating effect of this secondary bile acid on the expression of BCAC in comparison with ACF was investigated by us. In this study, expression of ACF was markedly inhibited by exposure of cholic acid whereas that of BCAC was slightly promoted. Furthermore, number of crypts / focus and diameter of ACF was decreased by cholic acid, yet the number and size of BCAC were increased by the bile $\operatorname{acid}^{22}$. These results indicate a clear difference on the biological properties of both lesions and again support that BCAC are probably the genuine premaligant lesions for colorectal cancers.

\section{Induction of Cellular Apoptosis in BCAC and Its Significance}

It seems to be true that there are multiple mechanisms for the actions of chemopreventive agents. Induction of cellular apoptosis is regarded as one of important mode of actions of them. This biological action is considered to be particularly important for the non-steroidal antiinflammatory drugs (NSAIDs) among a variety of chemopreventive compounds. In fact, it is known that some NSAIDs induce cellular apoptosis ${ }^{23}$. However, such evidences of NSAIDs have been obtained mostly in vitro studies. Thus, it is noteworthy to know in in vivo studies whether such agents induce apoptosis in the premalignant lesions or not. A NSAID (sulindac) was investigated for the potential to generate apoptosis in the precancerous lesions (BCAC and ACF) for colorectal cancers by us. Exposure of sulindac in diet (200 or $400 \mathrm{ppm})$ dose-dependently increased the apoptotic index (TUNEL staining was used as a biomarker for the apoptosis) in BCAC. However, no significant increase of the index was confirmed in the case of ACF (Table 1). In this study, the degree of sulindac-induced reduction of cell proliferation in $\mathrm{BCAC}$ exceeded that seen in $\mathrm{ACF}^{24}$.

\section{Comments}

Data provided by us suggest that BCAC are a possibly direct precursor for colorectal cancers. We would like emphasis that BCAC are promising biomarker for the risk assessment of environmental carcinogens and also for screening of chemopreventive agents, although the bio-assay for BCAC is technically more difficult than for ACF. The finding of $\mathrm{BCAC}$ will provide an important clue for the understanding of premalignant lesions for the large bowel cancers and the mechanisms of intestinal carcinogenesis. Our recent results from the comparative analysis on BCAC and colon cancers in rats suggest that $\beta$-catenin mutation is selected during the colon carcinogenesis. Recently, Paulsen et al. ${ }^{25}$ reported the absence of "classical" ACF in the colon of Min/+ mice. Instead, they identified the flat dysplastic lesions, which were denoted as ACFMin. Such lesions with
Table 1. Apoptotic Index of Normal Crypts, ACF and BCAC

\begin{tabular}{cccc}
\hline Groups (Treatment) & Normal crypts & ACF & BCAC \\
\hline AOM + basal diet & $0.11 \pm 0.10^{\mathrm{a}}$ & $0.17 \pm 0.60$ & $0.15 \pm 0.55$ \\
& $(16)$ & $(223)$ & $(56)$ \\
$\mathrm{AOM} \rightarrow$ sulindac & $0.15 \pm 0.12$ & $0.20 \pm 0.72$ & $0.99 \pm 1.43^{\mathrm{b}}$ \\
$(200$ ppm $)$ & $(16)$ & $(212)$ & $(56)$ \\
AOM $\rightarrow$ sulindac & $0.17 \pm 0.10$ & $0.25 \pm 0.66$ & $1.45 \pm 2.71^{\mathrm{b}}$ \\
$(400 \mathrm{ppm})$ & $(16)$ & $(166)$ & $(69)$ \\
\hline
\end{tabular}

Numbers in parenthese are numbers of examined lesions. ${ }^{a}$ : Mean \pm SD. ${ }^{\mathrm{b}}$ : Significantly different from the group with $\mathrm{AOM}+$ basal diet $(\mathrm{P}<0.0003)$.

flat structures are hidden in the surrounding mucosa. These evidences are consistent with the results on BCAC in our studies. Although intestinal lesions like BCAC have not yet been clarified in humans, it is likely that similar types of mutations may occur in the early appearing lesions and tumors in the colons of humans.

\section{References}

1. Bird R. Observation and quantification of aberrant crypts in the murine colon treated with a colon carcinogen: preliminary findings. Cancer Lett 1987; 37: 147-151.

2. Bird R, McLellan E, and Bruce W. Aberrant crypts, putative precancerous lesions, in the study of the role of diet in the aetiology of colon cancer. Cancer Surv 1989; 8: 189-200.

3. Bird R. Role of aberrant crypts foci in understanding the pathogenesis of colon cancer. Cancer Lett 1995; 93: 55-71.

4. Shivapurkar N, Tang Ferreira A, Nasim S, Garett C, and Alabaster O. Sequential analysis of k-ras mutations in aberrant crypt foci and colonic tumors induced by azoxymethane in Fischer-344 rats on high-risk diet. Carcinogenesis 1994; 15: 775-778.

5. Pretlow TP, Barrow B, Adshton WS, O'Riordan MA, Pretlow TG, Jurcisek JA, and Stellato TA. Aberrant crypts: Putative preneoplastic foci in human colonic mucosa. Cancer Res 1991; 51: 1564-1567.

6. Kawamori T, Tanaka T, Hara A, Yamahara J, and Mori H. Modifying effects of naturally occurring products on the development of colonic aberrant crypt foci induced by azoxymethane in F344 rats. Cancer Res 1995; 55: $1277-$ 1282.

7. Pretlow T, O'Riordan M, Somich G, Amini S, and Pretlow T. Aberrant crypts correlate with tumor incidence in F344 rats treated with azoxymethane and phytate. Carcinogenesis 1992; 13: 1509-1512.

8. Pretlow T, Cheyer C, and O'Riordan M. Aberrant crypt foci and colon tumors in F344 rats have similar increases in proliferative activity. Int J Cancer 1994; 56: 599-602.

9. Zheng Y, Kramer P, Lubet R, Steele V, Kellof G, and Pereira M. Effect of retinoids on AOM-induced colon cancer in rats: modulation of cell proliferation, apoptosis and aberrant crypt foci. Carcinogenesis 1999; 20: 255-260.

10. Rao C, Wang C, Smith B, Lubet R, Kellof G, Steele V, and Reddy B. Enhancement of experimental colon cancer by genistein. Cancer Res 1997; 57: 3717-3722. 
11. Yamada Y, Yoshimi N, Hirose Y, Kawabata K, Matsunaga $\mathrm{K}$, Shimizu M, Hara A, and Mori H. Frequent $\beta$-catenin gene mutations and accumulations of the protein in the putative preneoplastic lesions lacking macroscopic aberrant crypt foci appearance, in rat colon carcinogenesis. Cancer Res 2000; 60: 3323-3327.11.

12. Yamada Y, Yoshimi N, Hirose Y, Matsunaga K, Katayama M, Sakata K, Shimizu M, Kuno T, and Mori H. Sequential analysis of morphological and biological properties of $\beta$ catenin-accumulated crypts, probable premalignant lesions independent of aberrant crypt foci in rat colon carcinogenesis. Cancer Res 2001; 61: 1874-1878.

13. Pretlow T, O'Riordan M, Spancake K, and Pretlow T. Two types of putative preneoplastic lesions identified by hexosaminidase activity in whole-mounts of colons from F344 rats treated with carcinogen. Am J Pathol 1993; 142: 1695-1700.

14. He T, Sparks A, Rago C, Hermeking H, Zawal L, da Costa L, Mori P, Vogelstein B, and Kinzler K. Identification of cMYC as a target of the APC pathway. Science 1998; 281: $1509-1512$.

15. Tetsu O and McCormik F. 66-Catenin regulates expression of cyclin D1 in colon carcinoma cells. Nature 1999; 398: 422-426.

16. Ikeda S, Kishida S, Yamamoto H, Murai H, Koyama S, and Kikuchi A. Axin, a negative regulator of the Wnt signaling pathway, forms a complex with GSK- $3 \beta$-and $\beta$-catenin and promotes GSK-3 $\beta$-dependent phosphorylation of $\beta$-catenin. EMBO J 1998; 17: 1371-1384.

17. Korineck V, Barker N, Morin P, van Wichen D, de Weger, R, Kinzler K, Vogelstein B, and Clevers H. Constitutive transcriptional activation by a $\beta$-catenin-Tcf complex in APC-/- colon carcinoma. Science 1997; 275: 1784-1787.

18. Kawamori T, Rao C, Seibert K, and Reddy BS Chemopreventive activity of celecoxib, a specific cyclooxygenase-2 inhibitor, against colon carcinogenesis. Cancer Res 1998; 58: 409-412.

19. Reddy BS, Hirose Y, Lubet R, Steele V, Kellof G, Paulson S, Seibert K, and Rao C. Chemoprevention of colon cancer by specific cyclooxigenase-2 inhibitor, celecoxib, administered during different stages of carcinogenesis. Cancer Res 2000; 60: 293-297.

20. Yamada Y, Yoshimi N, Hirose Y, Hara A, Shimizu M, Kuno T, Katayama M, Qiao Z, and Mori H. Suppression of occurrence and advancement of $\beta$-catenin-accumulated crypts, possible premalignant lesions of colon cancer; by selective cyclooxygenase-2 inhibitor, celecoxib. Jpn J Cancer Res 2001; 92: 617-623.

21. Magnusen BA and Bird R. Reduction of aberrant crypt foci induced in rat colon with azoxymethane or methylnitrosourea by feeding cholic acid. Cancer Lett 1993; 68: $15-23$.

22. Hirose Y, Kuno T, Yamada Y, Sakata K, Katayama M, Yoshida K, Qiao Z, Hata K, Yoshimi N, and Mori H. Carcinogenesis 2002; Azoxymethane-induced $\beta$-cateninaccumulated crypts in colonic mucosa of rodents as an intermediate biomarker for colon carcinogenesis. Carcinogenesis 2002; 23 (in press).

23. Tsujii $\mathrm{M}$ and Dubois RN. Alterations in cellular adhesion and apoptosis in epithelial cells overexpressing prostaglandin endoperoxide synthase 2. Cell 1995; 83: 493501.

24. Kuno T, Yamada Y, Hirose Y, Katayama M, Sakata K, Hara A, Saji S, and Mori H. Induction of apoptosis by sulindac in azoxymethane-induced possible colonic premalignant lesions in rats. Jpn J Cancer Res 2002; 93: 242-246.

25. Paulsen JE, Steffensen IL, Loberg EM, Husoy T, Mamork E, and Alexander J. Qualitative and quantitative relationship between dysplastic aberrant crypt foci and tumorigenesis in the Min/+ mouse colon. Cancer Res 2001; 61: 5010-5015. 